\title{
Contribuciones de Zamorano a la Acuacultura Latinoamericana
}

\author{
Daniel E. Meyer ${ }^{1}$
}

Resumen. Las actividades de acuacultura en Zamorano comenzaron en 1976, cuando se sembraron 400 ejemplares de la tilapia de Java (Tilapia mossambica, ahora Oreochromis mossambicus) en un estanque construido para este propósito. Desde entonces, Zamorano ha contribuido a promover la acuacultura al capacitar personas interesadas en cultivar peces y crustáceos, desarrollando las instalaciones físicas adecuadas y realizando múltiples y variados trabajos de investigación y extensión. Las contribuciones y logros más significativos de Zamorano en el área de la acuacultura son haber capacitado a más de 7,000 personas en los fundamentos de la acuacultura y la distribución de más de 10,000 ejemplares de varios manuales técnicos sobre temas de la acuacultura y cultivo de tilapia. Técnicos de Zamorano han impartido cursos cortos sobre estos temas en 10 países, desde 1990. La investigación acuícola en Zamorano se ha enfocado en el uso de la hormona 17-Alfa-MetiloTestosterona para controlar el sexo de alevines de tilapia, el desarrollo de una infraestructura y manejo adecuados para los peces reproductores, el uso eficiente del alimento concentrado, la adaptabilidad de la tilapia al agua salina y salobre, y estudios ambientales.

Palabras clave: Capacitaciones técnicas, piscicultura, reversión sexual, tilapia.

\section{Contributions of Zamorano to Aquaculture in Latin America}

\begin{abstract}
Activities in aquaculture were begun in Zamorano in 1976 with the stocking of 400 fingerlings of the Java tilapia (Tilapia mossambica, now Oreochromis mossambicus) in a pond recently constructed for this purpose. Since then Zamorano has contributed to promoting aquaculture by training persons interested in farming fish and crustaceans, developing appropriate infrastructure and through varied and frequent research and extension activities. The most significant Zamorano accomplishments in aquaculture have been to train more than 7000 persons on the fundamentals of aquaculture and the distribution of more than 10,000 copies of several technical manuals on the topics of aquaculture and culture of tilapia. Technicians from Zamorano have offered short courses on these topics in ten different countries since 1990. Research efforts in aquaculture have focused on the utilization of the hormone 17-Alpha-Methyl-Testosterone in controlling the sex of tilapia fry, developing the infrastructure and management protocols for tilapia broodstock, efficient use of feeds and feeding fish and shrimp, the adaptability of tilapia to saline and brackish water, and environmental studies.
\end{abstract}

Keywords: Fish culture, technical training, tilapia, sex reversal.

\section{Introducción}

El primer intento. En noviembre de 1954, el Dr. Wilson Popenoe recibió en la Escuela Agrícola Panamericana (EAP), Zamorano, Honduras, al Dr. S.Y. Lin de China-Taiwán, un especialista en la pesca continental y la piscicultura dulceacuícola. La visita del Dr. Lin fue parte de su trabajo patrocinado por la FAO para evaluar, durante cinco meses, el potencial para la pesca en los cuerpos de aguas continentales en Honduras y para desarrollar la piscicultura en diferentes partes del país (Lin 1954).

Durante sus conversaciones, el Dr. Popenoe expresó su interés en establecer un centro de capacitación en piscicultura en Zamorano. El Dr. Lin recomendó a Popenoe enviar a un recién graduado de Zamorano al Instituto Politécnico de Alabama en Auburn, Alabama, Estados Unidos, para estudiar piscicultura durante unos dos años. Luego, esta persona podría regresar a Honduras para coordinar el centro y entrenar a otros. El Dr. Popenoe contemplaba la construcción de una estación piscícola en Zamorano con instalaciones para la reproducción y cría de peces para ser distribuidos entre los piscicultores de la región. Lamentablemente, y por alguna razón desconocida hasta ahora, el Dr. Popenoe nunca mandó a nadie a estudiar piscicultura. En su carta informativa de diciembre de 1955, Popenoe menciona que un

\footnotetext{
${ }^{1}$ Escuela Agrícola Panamericana, Zamorano, Honduras, correo electrónico dmeyer@zamorano.edu
} 
estanque en propiedad de Zamorano fue sembrado con ejemplares de la carpa común, peces obtenidos con la ayuda del Dr. Lin.

El Dr. Albert S. Muller, director de la EAP de 1964 a 1968, quiso continuar con los esfuerzos de Wilson Popenoe para desarrollar la acuacultura en Zamorano. Él mandó a construir el Lago de Monte Redondo y lo sembró con ejemplares de carpa para la pesca deportiva.

Segundo intento. Fue hasta en 1975, con el Sr. Kermit H. Adams, Director de Zamorano, que surgió de nuevo la idea. El Sr. Adams invitó al campus de Zamorano a un representante de una ONG de Tegucigalpa para ofrecer una charla sobre la piscicultura de tilapia. La ONG había empezado a promover la piscicultura a pequeña escala entre las familias del área rural de Honduras, empleando una metodología sencilla desarrollada en Sur América. Asistieron a la charla el Sr. Adams, algunos alumnos y varios docentes. Al concluir la presentación, el Director habló conmigo, como biólogo de Zamorano, para motivarme a iniciar trabajos de piscicultura en Zamorano, utilizando la tilapia como especie a cultivar.

\section{Logros en Acuacultura}

La construcción de algunos estanques se inició en 1976 en el lugar El Trapiche, cerca del Lago de Monte Redondo, en Zamorano, los cuales fueron sembrados con alevines de la tilapia de Java (O. mossambicus). Ese mismo año se estableció el módulo de acuacultura (Figura 1) y hasta 1987, se impartió un curso teórico formal sobre los fundamentos de la acuacultura a todos los alumnos de segundo año.

Este curso de acuacultura quedó como parte del programa de estudio en zootecnia en el Programa de Ingeniería Agronómica (PIA) iniciado en 1987, y más reciente como curso obligatorio para los alumnos del Departamento de Ciencia y Producción Agropecuaria (CPA) en el programa de estudio conocido como $4 \times$ 4, iniciado en 1999.

Las instalaciones acuícolas de Zamorano (Figura 2) incluyen 17 estanques excavados en la tierra con un total de $11,000 \mathrm{~m}^{2}$ de espejo de agua (Figura 2, \#3). Hay 29 pilas de concreto con un área superficial de
$270 \mathrm{~m}^{2}$ y seis tanques circulares $\left(140 \mathrm{~m}^{3}\right.$ de capacidad cada uno) montados sobre el terreno (Figura 2 \#5). Se construyó en el 2003 un invernadero de $620 \mathrm{~m}^{2}$ que alberga tres tanques con revestimiento de plástico con un total de $210 \mathrm{~m}^{3}$ de capacidad (Figura $2 \# 2$ ), seis tanques de fibra de vidrio (1500 $\mathrm{L}$ de capacidad cada uno) y una pila de concreto de $5 \times 12 \mathrm{~m}$ (Figura 4). Además, en el Lago de Monte Redondo (Figura 2 \#4) hay unas 36 jaulas de varias dimensiones para el engorde y cultivo experimental de peces.

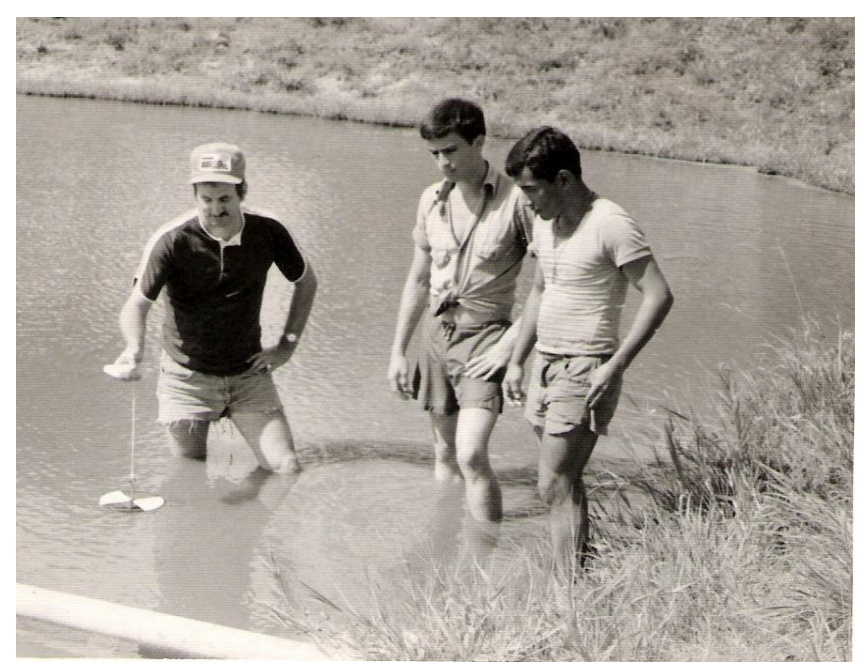

Figura 1. Daniel Meyer, acuacultor pionero en Zamorano, enseñando en el módulo de acuacultura a dos estudiantes de Zamorano, c1980.

El Laboratorio de Acuacultura de la Escuela Agrícola Panamericana, Zamorano, ha sido desarrollado como un centro de capacitación. Tiene varios edificios y equipos básicos para facilitar las tareas y actividades del aprender-haciendo de los alumnos quienes realizan sus trabajos prácticos atendiendo a los cultivos de peces y crustáceos en el laboratorio (Figura 3).

Los alumnos de Zamorano capacitados en los fundamentos de la acuacultura han tenido la oportunidad de trabajar con varias especies de peces y crustáceos, además de la tilapia de Java. A continuación el nombre científico, nombre común y fecha de introducción a Zamorano para cada una: 
Peces (tilapias):

- Oreochromis mossambicus (tilapia de Java) 1976

- O. niloticus (tilapia del Nilo) 1979

- O. urolepis hornorum (tilapia de Wami) 1983

- O. aureus (tilapia azul) 2012

Otros peces:

- Cyprinus carpio (carpa común) 1955

- Ctenopharyngodon idella (carpa herbívora o carpa grama) 1978

- Colossoma macropomum (tambaquí) 1985

Crustáceos:

- Cherax quadricarinatus (langosta australiana de agua dulce) 1997

- Litopenaeus vannamei (camarón blanco del Pacífico) 1990

- Macrobrachium rosenbergii (langostino de río de Malasia) 1978

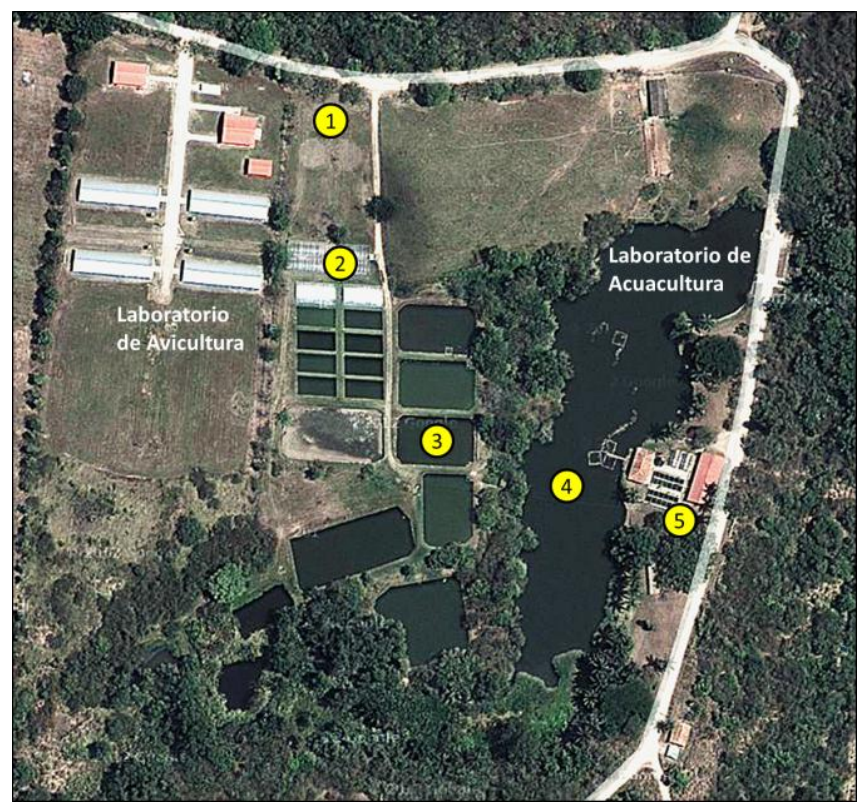

Figura 2. Vista aérea del Laboratorio de acuacultura en Zamorano, Hondura, en marzo del 2011, muestra la infraestructura para enseñar los fundamentos de la acuacultura. 1) inicio de la construcción de los tanques circulares, 2) invernaderos, 3) lagunas, 4) lago de Monte Redondo con jaulas para cultivar peces, y 5) laboratorios, salones de enseñanza y pilas de concreto (foto tomada de Google Earth).

\section{Proyección de Zamorano al Cultivo de Tilapia}

La Escuela Agrícola Panamericana, Zamorano, a contribuido bastante a la enseñanza, investigación y transferencia de las técnicas y producción acuícola en Honduras, en la región centroamericana y en el mundo. A continuación una breve descripción de algunas de las contribuciones más importantes.

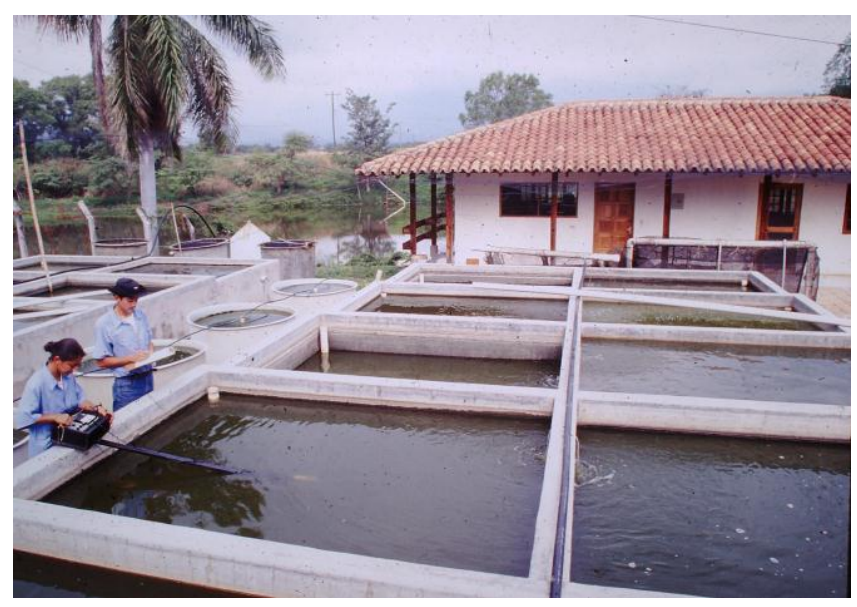

Figura 3. Estudiantes de Zamorano en prácticas del aprender haciendo, están monitoreando el agua para determinar la calidad para la producción acuícola.

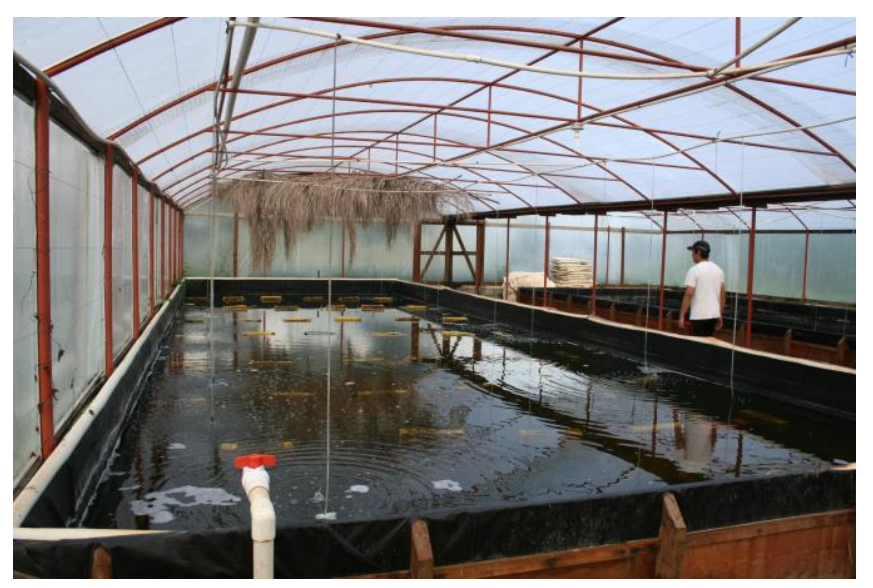

Figura 4. Tanque de $12 \times 5 \mathrm{~m}$ dentro del invernadero de $620 \mathrm{~m}^{2}$ construido en 2003 para tener mejor control de la temperatura del agua en Zamorano.

Curso teórico en programa formal educativo de 
Zamorano. Desde 1976, más de 4,000 alumnos del Programa de Agrónomo y de Ingeniero Agrónomo han sido capacitados en los fundamentos del cultivo de peces y especies de crustáceos en el curso teórico de acuacultura/producción acuícola. Desde 1976 al 2012, todos los alumnos de la institución realizan 60 horas de prácticas en acuacultura como parte de su capacitación en el aprender-haciendo.

Graduados de Zamorano en el sector privado y público. Varios graduados de Zamorano se han didicado a la acuacultura y han alcanzado puestos importantes en la acuacultura en el sector privado y público (Figura 5).

Publicación de Materiales Educativos y Didácticos. Con el apoyo financiero de múltiples fuentes, Zamorano ha publicado varios manuales sobre diversos temas de la acuacultura y ofrecido capacitaciones a personas interesadas en muchos países. La publicación de estos materiales didácticos y los viajes realizados han sido financiados por las donaciones y contribuciones de varias agencias del gobierno de los Estados Unidos de América, España, Alemania, El Salvador y varias agencias privadas.

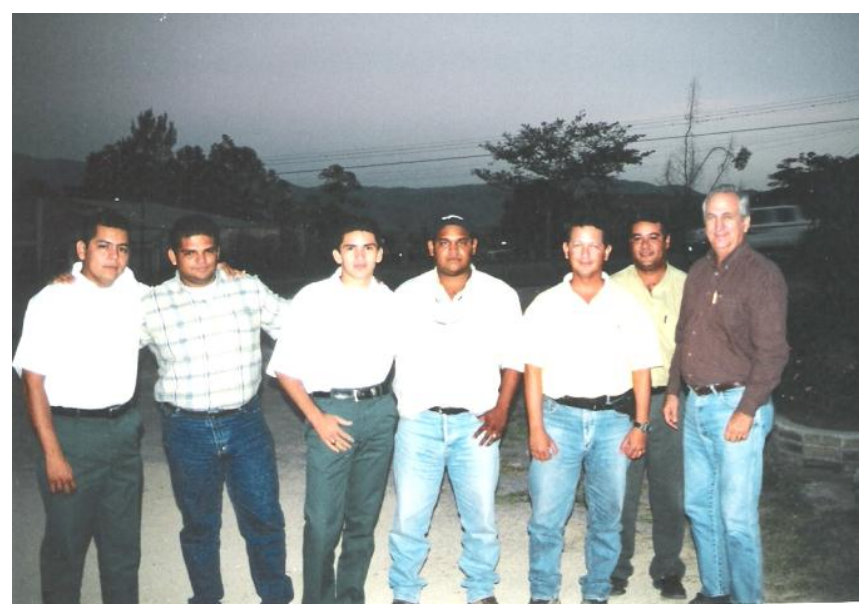

Figura 5. El Dr. Daniel Meyer y algunos de sus exestudiantes graduados en Zamorano quienes han desarrollado su carrera profesional en la acuacultura.

Cuerpo de Paz en Honduras. Se publicó un plan de trabajo para los voluntarios del Cuerpo de Paz en Honduras en el área de la piscicultura (Meyer 1986) con lineamientos para su desempeño en promover el cultivo de tilapia en zonas rurales de Honduras.

Introducción a la Acuacultura. Este libro es un compendio de los temas incluidos en el curso de acuacultura desarrollado durante los últimos 35 años en Zamorano. El manual, de 159 páginas, ha sido publicado varias veces, la última en 2008 (Meyer 2008). Se estima que 5,000 ejemplares de esta publicación han sido distribuidos desde 1990 al 2012.

Manual de Prácticas en Acuacultura. Este manual describe las prácticas incluidas en el aprender haciendo en acuacultura que cada alumno realiza en su programa formal educativo para optar a su título en Zamorano. Se ha distribuido más de 2,000 ejemplares del manual a los alumnos del programa formal académico en Zamorano (Meyer et al. 2003). La última revisión del manual fue en 2011 y está disponible ahora solamente en formato electrónico.

\section{Manual Práctico: Reproducción y Cría de Alevines} de Tilapia. Este manual resume las instalaciones, técnicas y manejos requeridos para la producción moderna de alevines machos de tilapia con el uso de la hormona Alfa-17-Metilo-Testosterona para la reversión sexual. El manual ha sido traducido al inglés y francés, y hasta el 2012 se han distribuido unos 700 ejemplares en Centro América (Meyer y Triminio Meyer 2007)..

Cultivo de Tilapia en Fincas Integradas Utilizando Insumos de Bajo Costo: Manual para Productores y Productoras. Esta publicación está dirigida a familias del área rural con interés en cultivar tilapia con un mínimo de insumos comprados. Se ha distribuido unas 3,000 ejemplares de este manual (Meyer et al. 2003)..

Acua-Noticias Zamorano. Desde 1996 al 2002, se publicaron varios números de una hoja informativa sobre la acuacultura como parte de las actividades de proyección acuícola en Zamorano. En total se distribuyeron unas 2,000 ejemplares de estas hojas.

Sitio web www.acuacultura.org. Zamorano participó 
desde 1999 al 2007 en el Programa Colaborativo de Apoyo a la Investigación en Acuacultura (CRSP en Acuacultura) con fondos de la USAID en Washington. Como parte del programa CRSP, se hizo en 2003 el sitio web con información en español para las personas interesadas en aprender los fundamentos del cultivo de tilapia y otras especies. El sitio web continúa funcionando y provee información a los interesados de Latinoamerica.

Cursos sobre los fundamentos de la producción acuícola. Desde 1990 al 2012, personal de Zamorano ha ofrecido cursos cortos sobre los fundamentos de la producción de peces y crustáceos cultivados en 10 países de Latinoamérica (Belice, Chile, Colombia, Costa Rica, El Salvador, Guatemala, Honduras, Nicaragua, Panamá y República Dominicana). Estos cursos se realizaron con financiamiento del Programa Colaborativo en Apoyo a la Investigación en la Acuacultura (A/CRSP) de la USAID, del gobierno de los Estados Unidos y otras fuentes. Se logró capacitar a más de 3,000 personas en cursos tpipicamente de tres días duración.

Cursos con profesionales Latinoamericanos. Con fondos de la DSE (Deutsche Stiftung für Internationale Entwicklung; Agencia de Desarrollo Internacional) de Alemania, durante el periodo de 1992 a 1997 se realizó anualmente una capacitación de profesionales latinoamericanos en los fundamentos de la acuacultura. Cada capacitación duró seis meses e incluía un fuerte componente de aprender-haciendo, trabajos de investigación, viajes y giras a otros países. Se capacitaron 12 profesionales en el programa.

Eventos técnicos/científicos. La participación de Zamorano en reuniones técnicas para aprender y transmitir conocimientos ha sido importante. Algunos de los eventos en que ha participado son:

- Zamorano participó en el Primer Encuentro Nacional de Piscicultura celebrado en Comayagua, Departamento de Comayagua, Honduras, los días 11 y 12 de octubre de 1985 (Meyer 1986).

- Se organizó y se llevó a cabo el Encuentro Nacional de Acuicultura en 1988 en Zamorano con la participación de más de 100 personas.
Se publicó la memoria del evento en la revista Ceiba (Meyer 1989; Meyer et al. 1989).

- Desde 1991, Zamorano ha tenido una participación activa y amplia (Meyer 2001a; Meyer 2001b) en todos los Simposios Centroamericanos de Acuacultura, eventos organizados por la Asociación Nacional de Acuicultores de Honduras (ANDAH).

- Personal de acuacultura de Zamorano ha participado en reuniones técnicas/científicas internacionales en: Brasil, Corea del Sur, Costa Rica, Côte d' Ívoire, Estados Unidos, España, Filipinas, Ghana, Guatemala, Italia, Kenia, México, Polonia, República de África del Sur, República Popular China, Tailandia y Venezuela.

\section{Investigación y Desarrollo de Técnicas Nuevas}

Uso de la hormona 17-Alfa-Metilo-Testosterona. Zamorano fue la primera estación piscícola en Honduras en utilizar la hormona 17-Alfa-MetiloTestosterona, un esteroide anabólico, para definir sus efectos sobre alevines de tilapia (Meyer 1990, Meyer and Smitherman 1993). La hormona es suministrada en el alimento concentrado y causa dos efectos importantes. Primero, el efecto anabólico del esteroide que acelera su crecimiento. Segundo, la hormona causa una reversión sexual resultando en poblaciones de solamente machos. Ambos efectos son beneficiosos porque los machos de tilapia ganan peso más rápidamente que las hembras.

En un estudio se trataron los alevines para evaluar los efectos de la hormona en la ganancia de peso durante 180 días de cultivo. Las poblaciones tratadas eran de alevines de sexos mezclados, de solamente machos genéticos (híbridos machos obtenidos por cruzar O. niloticus $\odot \times$ O. urolepis hornorum $\bigcirc^{\widehat{)}}$ ) o de solamente hembras genéticas (obtenidas por cruzar una hembra con un macho fenotípico, pero de genotipo femenino). En general, los peces tratados con 17-Alfa-Metilo-Testosterona lograron, a los 30 días de cultivo, un peso $12 \%$ mayor que los no tratados. La diferencia desapareció después de 5 meses de engorde.

Se determinó que el tratar peces con 17-AlfaMetilo-Testosterona aumentan los procesos anabólicos 
resultando en una tasa de ganancia de peso $\pm 12 \%$ mayor comparado con los peces no tratados. Se infirió que el efecto de la reversión sexual de la hormona aumenta la ganancia de peso en $\pm 30 \%$ en los machos de tilapia tratados. Así, cuando se tratan los alevines de tilapia con la hormona para producir poblaciones de solamente machos, la hormona aumenta la tasa de ganancia de peso de 33 a $42 \%$, comparado con los alevines no tratados.

Infraestructura y manejo de los peces reproductores. Con múltiples trabajos de tesis realizados desde el 2002, Zamorano ha desarrollado una infraestructura y un manejo de los peces adultos para incrementar la producción de crías y sostener está producción durante los 12 meses del año. Algunas de las investigaciones son las detalladas a continuación.

En unos estudios se comparó la producción de crías en pilas con o sin una cubierta de plástico de polietileno usadas en invernadero (Ballesteros Ferrel 2001; Paucar Nieto 2002). La cubierta de plástico aumentó $3^{\circ} \mathrm{C}$ la temperatura del agua, comparado con las pilas sin cubierta, lo que se tradujo en una mayor producción de crías. Luego se compararon varios ambientes para la producción de crías de tilapia en una finca comercial en el norte de Honduras. Pero, no hubo diferencia en la producción total de alevines con los adultos en estanques revestidos de plástico, concreto o de tierra (Quan García 2000).

En un estudio de producción de alevines de tilapia, la mayor producción de alevines aptos para la fase de reversión sexual ( $\leq 14 \mathrm{~mm}$ de largo) fue lograda capturando los alevines un día de por medio a partir de los 14 días después de la siembra de los peces adultos (Galindo Pineda 2000).

La remoción de las crías de tilapias de la cavidad bucal de la hembra es una tarea tediosa y a veces delicada. Para mejorar este trabajo, se comparó la confinación de los peces adultos en bolsas de malla para facilitar la captura de las hembras y la remocíon de las crias de su cavidad bucal, con peces libres en las pilas. Se logró aumentar $400 \%$ la producción de crías con los reproductores confinados (Araúz Méndez 2008).

Las unidades de producción de crías y el sistema de incubación de los huevos, embriones y peces larvas de tilapia, son elementos importantes usados diariamente en la capacitación de los alumnos de la institución y cursillistas quienes tienen interés en aprender los fundamentos de la piscicultura y el manejo comercial de cultivos de tilapia (Bowman et al. 2008; Martínez et al. 2005; Meyer y Triminio Meyer 2007).

Alimento concentrado y alimentación. Usualmente, el alimento concentrado es el insumo más caro en el engorde de peces. Su alto costo se debe a su alto contenido de proteína cruda y porque su transformación en perdigones flotantes es costosa. Se realizó una serie de investigaciones para lograr un mejor uso del alimento concentrado para engordar tilapia en Zamorano.

Se alimentó la tilapia en peceras de vidrio con la cantidad diaria de concentrado ofrecido en una, dos o cuatro porciones (Caamaño Reyes 1998). El consumo del alimento incrementó con el número de porciones diarias. En general, el pez ganó más peso diario al consumir más alimento. El consumo típico era alto un día y bajo al día siguiente.

Se probó ofrecer alimento concentrado a los peces cada día y un día de por medio en estanques de $200 \mathrm{~m}^{2}$ de espejo de agua. Comenzando con alevines de $1 \mathrm{~g}$ de peso promedio y a lo largo de 5 meses de engorde, el manejo con restricción en el uso del alimento resultó en una biomasa final de peces igual al uso diario del alimento. Los ingresos netos (ingresos totales/costos totales $\times 100$ ) al ofrecer alimento diariamente o día de por medio fueron de 19 y $34 \%$, respectivamente (Barragán Zambrano 2006).

Se combinó el engorde de peces adultos con el preengorde de alevines, buscando alternativas para hacer más eficiente la producción y uso del alimento concrentrado y otros insumos. Se engordaron peces en jaulas colocadas en un estanque de $1000 \mathrm{~m}^{2}$ de superficie y con 3000 alevines. Los peces en las jaulas recibían concentrado y los alevines crecieron consumiendo los desperdicos del engorde y estiércol (1.5 kg materia seca/día) de una porqueriza. Con este manejo se logró un Índice de Conversión Alimentica (ICA) de 0.76:1.00 y una rentabilidad (ventas - costos totales/costos totales $\times 100$ ) de $40 \%$ (Suazo Zepeda 2002; Trejo Ortega 2002).

Usando gallinaza, alimento concentrado y los desperdicios del engorde de peces en jaulas, se 
preengordaron lotes de 5000 alevines en tanques con geomembrana de $12.4 \mathrm{~m}$ de diámetro y $1.2 \mathrm{~m}$ de profundidad. Con la gallinaza fresca de ponedoras se logró mayor producción con menores costos en el preengorde de alevines de tilapia en ciclos de 28 días.

Se logró una producción integrada de tilapia con lechugas probando exitosamente sistemas de acuaponía. Combinando la producción de peces con lechugas resultó en un 100\% de sobrevivencia, y lechugas completamente limpias y sin daños físicos (Arriaza Casteñeda y Martínez Cabrera 2009; Grande Zometa y Luna Vega 2010).

Adaptaciones a la salinidad. La tilapia es un pez eurihalino que se adapta a altas concentraciones de sal en el agua. Se realizaron ensayos determinando la tolerancia que tiene la tilapia del Nilo y roja a varias salinidades del agua. El 100\% de los alevines de 1 día de edad, de la tilapia del Nilo y roja, sobrevivieron el pasar directamente del agua dulce a agua con 20,000 ppm de sal. Con salinidades mayores de $25,000 \mathrm{ppm}$, la mayoría de los alevines de tilapia del Nilo murieron. La aclimatación de los alevines de la tilapia del Nilo ayudó en su sobrevivencia a salinidades mayores de $20,000 \mathrm{ppm}$. En otro estudio, la mayor producción de crías de los adultos de tilapia roja fue con 0 o 10,000 ppm de sal en el agua. Con salinidades de 20,000 y 30,000 ppm, la producción de crías fue menor (Tecún Díaz 2012).

Estudios ambientales. Zamorano participó en un estudio de la calidad del agua en dos esteros en la zona sur de Honduras para evaluar los impactos del desarrollo del cultivo del camarón de mar en el Golfo de Fonseca. Se determinó que la mayoría de los contaminantes en los esteros eran generados por las comunidades y los animales criados y engordados dentro de las cuencas (Serrano Interiano 1996; Sampson Dávila 1997).

Se detectaron residuos de 11 pesticidas en muestras de agua, de sedimentos y biológicas, tomadas en ríos y esteros de la zona sur de Honduras. La mayoría de estos pesticidas estaban presentes a concentraciones menores de 0.1 ppm (Acevedo Estrada 1995).

Otras contribuciones. Se hizo un inventario de 20 fincas piscícolas para comparar las instalaciones y métodos usados en la producción y distribución de alevines de tilapia en Honduras (Triminio Meyer et al. 2007). Además, se desarrolló una hoja en Excel para ayudar en la planificación y construcción de estanques excavados en la tierra (Tollner et al. 2004). Con apoyo financiero del A/CRSP, se realizaron talles con ingenieros civiles para diseminar la información a usuarios potenciales en Honduras, Guatemala y Costa Rica.

\section{Conclusiones}

Desde 1976, Zamorano realiza actividades de acuacultura en su programa teórico formal educativo y en el aprender-haciendo. En 2012, el Laboratorio de Acuacultura de Zamorano cuenta con modernas instalaciones para llevar a cabo actividades relacionadas con el cultivo de varias especies de peces y crustáceos de importancia en la región Latinoamericana. Se han publicado varios manuales y folletos útiles para guiar a los piscicultores principiantes a tener éxito en el cultivo de tilapia. Se han impartido cursos cortos sobre los fundamentos del cultivo de tilapia y temas afines en 10 países, logrando capacitar a más de 7000 personas. Zamorano mantiene un sitio web www.acuacultura.org con información en español sobre el cultivo de tilapia y otros temas de interés y utilidad para apoyar a la acuacultura en Centro América y otras partes del mundo. La investigación acuícola en Zamorano se ha enfocado en las áreas del uso de la hormona 17-Alfa-MetiloTestosterona para controlar el sexo de alevines de tilapia, el desarrollo de una infraestructura y manejo adecuados para los peces reproductores, la utilización eficiente del alimento concentrado, la adaptabilidad de la tilapia al agua salina y salobre, y estudios ambientales. 


\section{Literatura Citada}

Acevedo Estrada, J.A. 1995. Monitoreo de pesticidas en agua de esteros del Golfo de Fonseca, Honduras. Proyecto Especial del Programa de Ing. Agr., Zamorano, Escuela Agrícola Panamericana, Honduras. $48 \mathrm{p}$.

Araúz Méndez, A. 2008. Comparación del manejo de los reproductores de tilapia roja en pilas de concreto con o sin hapas. Proyecto Especial del Programa de Ing. Agr., Zamorano, Escuela Agrícola Panamericana, Honduras. $10 \mathrm{p}$.

Arriaza Castañeda, A. y J.L. Martínez Cabrera. 2009. Producción hidropónica de lechuga integrada con el cultivo de tilapia con tres niveles de potasio y hierro. Proyecto Especial del Programa de Ing. Agr., Zamorano, Escuela Agrícola Panamericana, Honduras. $14 \mathrm{p}$.

Ballesteros Ferrel, J.M. 2001. Evaluación de la reproducción de tilapia del Nilo en pilas cubiertas con plástico. Proyecto Especial del Programa de Ing. Agr., Zamorano, Escuela Agrícola Panamericana, Honduras. $38 \mathrm{p}$.

Barragán Zambrano, D.I. 2006. Evaluation of the restricción of feed rations in Nile tilapia production (Oreochromis niloticus). Proyecto Especial del Programa de Ing. Agr., Zamorano, Escuela Agrícola Panamericana, Honduras. $15 \mathrm{p}$.

Bowman, J., A. Bart, R. Bolivar, W. Contreras-Sánchez, N. Gitonga, D. Meyer and H. Egna. 2008. A comparison of tilapia culture technologies: Linking research and outreach results across geographic regions. World Aquaculture 39(2): 39-44.

Caamaño Reyes, E.E. 1998. Relación entre la frecuencia de la alimentación y el consumo en tilapia (Oreochromis niloticus). Proyecto Especial del Programa de Ing. Agr., Zamorano, Escuela Agrícola Panamericana, Honduras. $17 \mathrm{p}$.

Galindo Pineda, J.C. 2000. Evaluación de dos sistemas de manejo para la producción de alevines de tilapia. Proyecto Especial del Programa de Ing. Agr., Zamorano, Escuela Agrícola Panamericana, Honduras. $15 \mathrm{p}$.

Grande Zometa, E.O. y P.R. Luna Vega. 2010. Comparación de la producción de lechuga a 6,12 y 18 plantas $/ \mathrm{m}^{2}$ con 40 y 70 ppm de nitrógeno total en acuaponía con tilapia. Proyecto Especial del Programa de Ing. Agr., Zamorano, Escuela Agrícola Panamericana, Honduras. $15 \mathrm{p}$.
Lin, S.Y. 1954. Personal correspondence from Dr. S.Y. Lin to Dr. Wilson Popenoe with the date of November 24th. Department of Fisheries, Food and Agriculture Organization (FAO) of the United Nations

Martínez, P.R., J. Molnar, E. Trejo, D. Meyer, S.T. Meyer and W. Tollner. 2005. Cluster membership as a competitive advantage in aquaculture development: a case study of tilapia producers in Olancho, Honduras. Journal of Aquaculture Economics \& Management 8(5/6): 281-294.

Meyer, D.E. 1986. Plan de trabajo en la piscicultura para los Voluntarios del Cuerpo de Paz en Honduras. Escuela Agrícola Panamericana, Tegucigalpa, Honduras. 50 p.

Meyer, D.E. 1986. Piscicultura en El Zamorano, p. 74-80. En: Memoria: Primer Encuentro Nacional de Piscicultura. Publicado por el Instituto Nacional Agraria, Tegucigalpa, Honduras.

Meyer, D.E. 1989. Realidad de la acuicultura en Honduras y sus limitaciones. Ceiba 30(2): 7-16.

Meyer, D.E. 1990. Growth, survival and sex-ratios of Oreochromis urolepis hornorum, $O$. niloticus and their hybrids $(O$. niloticus female $\times$ O.u.hornorum male) treated with 17- $\alpha$-methyl-testosterone. Ph.D. Dissertation, Department of Fisheries \& Allied aquacultures, Auburn University, Alabama, USA.

Meyer, D.E. 1995. Marine shrimp farming development in southern Honduras. Acta Hydrobiologica 37(1): 111120.

Meyer, D.E. 2008. Introducción a la Acuacultura. Publicado por la Escuela Agrícola Panamericana, Honduras. 159 p.

Meyer, D.E., N. Luna y G. Reyes (Eds.). 1989. Memoria: Encuentro Nacional de Acuicultura. Ceiba 30(2):1-84.

Meyer, D.E. (editor). 2001a. Proceedings of the Tilapia Sessions at the VI Central American Aquaculture Symposium. Associación Nacional de Acuicultores de Honduras (ANDAH), Tegucigalpa, Honduras. 148 p,

Meyer, D.E. 2001b. Nutrition and feeding of tilapia, pages 61-70. In: D.E. Meyer (editor). Proceedings of the Tilpia Sessions at the VI Central American Aquaculture Symposium. Associación Nacional de Acuicultores de Honduras (ANDAH), Tegucigalpa, Honduras.

Meyer, D.E., F. Martínez y S. Triminio Meyer. 2003. Producción de tilapia en fincas integradas utilizando insumos de bajo costo: manual para productores y productoras. Publicación del Programa Colaborativo en Apoyo a la Investigación en Dinámica de Estanques y Acuacultura (PD/A CRSP), USAID, Washington, D.C., EE.UU. 30 p. 
Meyer, D.E. and R.O. Smitherman. 1993. Growth, survival and sex-ratios of Oreochromis urolepis hornorum, $O$. niloticus and their hybrids (O. niloticus female $\times$ O.u.hornorum male) treated with 17-a.methyltestosterone, p. 62-71. In: R.S.V. Pullin, J. Lazard, A. Kothias and D. Pauley (eds). Third International Symposium on Tilapia in Aquaculture, Abidjan, Côte d' Ívoire. Published by the International Center for Living Aquatic Resources Management (ICLARM), Manila, The Philippines.

Meyer, D.E. y S. Triminio Meyer. 2007. Reproducción y Cría de Alevines de Tilapia: Manual Práctico. Publicación del Programa de Colaboración en Apoyo a la Investigación en Acuacultura (ACRSP), Universidad Estatal de Oregon, Corvallis, Oregon, EE.UU. 51 p.

Nuñez Cárdenas, C.S. 2012. Comparación del uso de gallinaza + urea, desperdicios del engorde de peces en jaulas y alimento concentrado para el pre-engorde de alevines de tilapia del Nilo (Oreochromis niloticus). Proyecto Especial del Programa de Ing. Agr., Zamorano, Escuela Agrícola Panamericana, Honduras. $21 \mathrm{p}$.

Paucar Nieto, F.P. 2002. Evaluación de cubiertas de plástico para la producción de alevines de tilapia (Oreochromis niloticus). Proyecto Especial del Programa de Ing. Agr., Zamorano, Escuela Agrícola Panamericana, Honduras. $15 \mathrm{p}$.

Quan García, V.L. 2000. Evaluación de la reproducción de tilapia (Oreochromis niloticus) en estanques revestidos de plástico, concreto y de tierra. Proyecto Especial del Programa de Ing. Agr., Zamorano, Escuela Agrícola Panamericana, Honduras. 18 p.
Suazo Zepeda, A.A. 2002. Cultivo combinado de tilapia (Oreochromis niloticus) en jaulas y alevines en un estanque integrado con credos. Proyecto Especial del Programa de Ing. Agr., Zamorano, Escuela Agrícola Panamericana, Honduras. 15 p.

Tecún Díaz, Y.M. 2012. Evaluación de la reproducción y crecimiento de tilapia roja (Oreochromis sp.) en agua a 0, 10,000, 20,000 y 30,000 ppm de sal en Zamorano. Proyecto Especial del Programa de Ing. Agr., Zamorano, Escuela Agrícola Panamericana, Honduras. $16 \mathrm{p}$.

Trejo Ortega, R.A. 2002. Cultivo combinado de tilapia (Oreochromis niloticus) en jaulas y alevines en un estanque bajo un manejo intensivo. Proyecto Especial del Programa de Ing. Agr., Zamorano, Escuela Agrícola Panamericana, Honduras. 16 p.

Triminio Meyer, S.A., J.J. Molnar, D.E. Meyer and E.W. Tollner. 2007. Tilapia fingerling production in Honduras. Journal of Applied Aquaculture 19(2):1-27.

Tollner, E.W., D. Meyer, S. Triminio-Meyer, B. Verma, G. Pilz and J.J. Molnar. 2004. Spreadsheet tools for developing surface water supplies for freshwater fish in developing countries. Journal of Aquaculture Engineering 31: 31-49.

Recibido para publicación el 6 de septiembre de 2012. Aceptado para publicación el 20 de enero de 2013. 